\title{
Escala de Autoeficácia para Autorregulação da Escrita: Adaptação e Validação para Universitários
}

\author{
Eduarla Resende Videira Emilio ${ }^{1}$ \\ Soely Aparecida Jorge Polydoro \\ Adriane Martins Soares Pelissoni ${ }^{2}$ \\ Marilda Aparecida Dantas ${ }^{2}$ \\ ${ }^{1}$ Instituto de Psicologia da Aeronáutica, Rio de Janeiro, Rio de Janeiro, Brasil \\ ${ }^{2}$ Universidade Estadual de Campinas, Campinas, São Paulo, Brasil
}

\begin{abstract}
Resumo
Neste estudo almejou-se traduzir, adaptar e buscar evidências de validade baseadas na estrutura interna e na relação com uma variável externa convergente para a Escala de Autoeficácia para Autorregulação da Escrita (EAARE) no contexto brasileiro. Trata-se de uma escala que avalia a percepção de capacidade do estudante em executar habilidades específicas de escrita. Participaram da pesquisa 430 universitários, que responderam à EAARE e a outra medida de aferição da crença de autoeficácia na escrita. A análise fatorial indicou a presença de uma única dimensão, cujo índice de consistência interna foi de 0,95. Em convergência com a literatura e com as expectativas formuladas, os resultados também apontaram uma relação estatisticamente significativa, forte e positiva entre os escores das duas escalas utilizadas. Desse modo, pode-se afirmar que a EAARE se constitui como um instrumento com evidências de validade e de fidedignidade apropriados para o uso no contexto brasileiro.

Palavras-chave: avaliação psicológica, linguagem escrita, redação científica, autopercepção, instrumentos de aprendizagem
\end{abstract}

\section{Self-Efficacy for Self-Regulated Writing Scale: Adaptation and Validation for Undergraduates}

\section{Resumen}

This validation study aimed at the translation, adaptation, and presentation of the Self-Efficacy Scale for Self-Regulated Writing (EAARE) in the Brazilian context based on its internal structure and relation with a convergent external variable. This scale assesses students' perception of their ability to perform specific writing skills. A total of 430 undergraduates participated in the study answering the EAARE and another writing self-efficacy assessment. The factor analysis indicated the presence of only one dimension with internal consistency of 0.95 and also a strong and positive relationship between the scores of the 2 scales applied in the study. These results are consistent with the literature and with the posited expectation; therefore it can be affirmed that EAARE is an instrument with proper evidence of validity and reliability to be used in the Brazilian context. Keywords: psychological assessment; written language; scientific writing; self-perception; learning tools.

\section{Escala de Autoeficacia para Autorregulación de la Escritura: Adaptación y Validación para Universitarios}

\begin{abstract}
Resumen
Se anheló en este estudio traducir, adaptar y buscar evidencias de validez, basadas en la estructura interior y en la relación con una variable exterior convergente, de la Escala de Autoeficacia para Autorregulación de la Escritura (EAARE) en el contexto brasileño. Se trata de una escala que evalúa la percepción de la capacidad del estudiante en ejecutar habilidades específicas de escritura. Participaron de la investigación 430 universitarios, que respondieron a la EAARE y la otra medida de evaluación de la creencia de autoeficacia en la escritura. El análisis factorial indicó la presencia de una sola dimensión, cuyo índice de consistencia interna fue 0,95 . En convergencia con la literatura y con las expectativas formuladas, los resultados también señalaron una relación estadísticamente significativa, fuerte y positiva entre las puntuaciones de las dos escalas utilizadas. De este modo, se puede afirmar que la EAARE se constituye como un instrumento con evidencias de validez y de fiabilidad apropiadas para el uso en el contexto brasileño.

Palabras clave: evaluación psicológica; lengua escrita; escritura científica; autopercepción; herramientas de aprendizaje.
\end{abstract}

\section{Introdução}

Os processos que os estudantes utilizam para regular a sua aprendizagem acadêmica têm sido objeto de pesquisas, com aumento de investigações sobre o tema (Balkis \& Duru, 2016; Carmo, 2017; Herndon \& Bembenutty, 2017; Marini \& Boruchovitch, 2014; Mello, Rosário, Polydoro, \& Högemann, 2018; Nava, 2017; Polydoro \& Emilio, 2017; Polydoro, Emilio, \&
Carmo, 2017; Polydoro \& Pedersen, 2017; Zimmerman \& Kitsantas, 2014). Embora existam modelos variados de autorregulação da aprendizagem, em linhas gerais, é consenso que ela abarca o controle dos processos cognitivos, motivacionais e comportamentais (Ganda \& Boruchovitch, 2018; Schunk \& Greene, 2018; Schunk \& Zimmerman, 1997; Zimmerman \& Schunk, 2011).

Entre os estudiosos do tema, há aqueles que enfocam nas estratégias de aprendizagem (metacognitivas 
e cognitivas) (Perassinoto, Boruchovitch, \& Bzuneck, 2013; Pozo, 1996), outros nas variáveis afetivas e motivacionais (Weiner, 2010; Wolters, 2011) e, ainda, aqueles que focam na tomada de decisão e nas ações dos estudantes autorregulados (Grau \& Whitebread, 2012; Volet, Vauras, \& Salonen, 2009). Na Teoria Social Cognitiva, o modelo autorregulatório de aprendizagem é caracterizado como um processo autodiretivo “(...) pelo qual os indivíduos consistentemente organizam e gerenciam seus pensamentos, emoções, comportamentos e ambiente para atingir objetivos acadêmicos." (Ramdass \& Zimmerman, 2011, p. 198). Portanto, os estudantes que autorregulam sua aprendizagem, controlam fatores potencialmente influentes para o sucesso em tarefas acadêmicas.

No conjunto das tarefas acadêmicas, uma delas se destaca em decorrência de sua importância como forma de registro, de organização do pensamento e de difusão de ideias e informações. Trata-se da tarefa de escrita. Esta apresenta desafios especiais para a autorregulação, uma vez que é tida como uma tarefa onerosa e longa, que requer, além de conhecimentos de vocabulário e gramática, altos níveis de regulação pessoal, para que sejam autoplanejadas, autoiniciadas e autossustentadas (Zimmerman \& Kitsantas, 2002; Zimmerman \& Risemberg, 1997). É plausível dizer que a autorregulação da escrita é imprescindível para o sucesso acadêmico, especialmente no Ensino Superior, afinal, espera-se que o estudante seja capaz de escrever utilizando vocabulários peculiares das diferentes áreas do conhecimento, de adequar a sua escrita aos diferentes gêneros de textos solicitados nas disciplinas (fichamento, resumo, artigo, resenha...) e de utilizar apropriadamente as citações de obras consultadas na preparação do texto (Nava, 2013; Nietto \& Alves, 2007). Ademais, espera-se que o estudante seja hábil para escolher estratégias de escrita adequadas aos seus objetivos, que elabore rascunhos de tópicos antes de começar a escrever, que monitore o seu desempenho e que revise e faça modificações nos seus textos de forma a manter a coerência e a clareza dele (Fernandes \& Frison, 2015; Granam, Harris, MacArthur, \& Santangelo, 2018; Nava, 2013; Thiede \& Bruin, 2018).

Zimmerman e Risemberg (1997) propõem um modelo de escrita constituído por três modos de autorregulação: ambiental, comportamental e pessoal. A autorregulação ambiental trata da estruturação de aspectos físicos e sociais que interferem na escrita. A autorregulação comportamental remete ao controle das atividades motoras associadas à escrita. Já a autorregulação pessoal refere-se à regulação de crenças cognitivas e estados afetivos ligados à escrita. Cada um desses modos triádicos de autorregulação interatua de modo interdependente, pois a autorregulação do ambiente influencia a autorregulação comportamental; a autorregulação comportamental afeta a autorregulação pessoal; e esta, por sua vez, gera impacto na autorregulação ambiental. Ademais, considerando as especificidades da tarefa de escrita, esses autores definiram a autorregulação da escrita como “(...) pensamentos, sentimentos e ações autogerados que os escritores usam para atingir vários objetivos literários, incluindo melhorar suas habilidades de escrita, bem como melhorar a qualidade do texto que criam." (Zimmerman \& Risemberg, 1997, p.76).

Nesse modelo autorregulatório da escrita, as crenças de autoeficácia para a escrita são tidas como um dos determinantes mais influentes dos processos que regem o curso da realização da composição textual (Zimmerman \& Bandura, 1994). Essas crenças são definidas como a confiança do indivíduo para realizar com êxito tarefas específicas de escrita (resumo, anotações, poesia etc.) ou para executar habilidades específicas de escrita (usar corretamente pontuações, redigir sem erros gramaticais, construir frases coerentes e coesas etc.) (Mccarthy, Meier, \& Rinderer, 1985; Pajares \& Johnson, 1994). A importância dessas crenças centra-se no seu papel mediador na relação entre as habilidades cognitivas e o próprio envolvimento do estudante (Pajares \& Johnson, 2007; Pajares \& Valiante, 1999).

Uma das pesquisas pioneiras sobre as crenças de autoeficácia para a escrita foi desenvolvida por Shell, Murph e Bruning (1989). Esses autores verificaram a capacidade preditiva das crenças de autoeficácia para tarefas de leitura e para tarefas de escrita, das crenças de expectativas de resultado sobre os desempenhos em leitura e em escrita. Os resultados da análise de regressão múltipla indicaram que as crenças de autoeficácia e de expectativas de resultado, em conjunto, explicaram $10 \%$ da variação do desempenho, sendo a autoeficácia o preditor mais forte. Portanto, as crenças de autoeficácia para a escrita tiveram um impacto direto sobre os resultados em escrita.

Pajares e Johnson (1994) também estudaram as relações entre a autoeficácia para escrita, expectativa de resultado, autoeficácia pessoal e desempenho em escrita de ensaios. Os resultados apontaram que a autoeficácia para escrita estava direta, moderada e significativamente relacionada com o desempenho em escrita. No modelo de regressão proposto pelos autores, as crenças de 
autoeficácia para escrita (especialmente as crenças em habilidades específicas de escrita) e a medida de desempenho anterior em escrita foram os únicos preditores significativos do desempenho em escrita, explicando $68 \%$ da variância dessa variável.

Em 1996, os mesmos autores testaram a influência das crenças de autoeficácia para escrita, da apreensão para escrever, da aptidão em escrita e do gênero sobre o desempenho em escrita de ensaios. Conforme hipotetizado, a aptidão e as crenças de autoeficácia para escrita dos alunos tiveram efeitos fortes, diretos e significativos no desempenho em escrita. Além disso, as crenças de autoeficácia para a escrita também influenciaram a apreensão, que, por sua vez, teve um impacto sobre o desempenho em escrita. $\mathrm{O}$ modelo testado representou 53\% da variância do desempenho em escrita (Pajares \& Johnson, 1996).

De modo semelhante, Pajares e Valiante (1999) notaram que a autoeficácia para a escrita foi a única variável motivacional que previu a variância da competência em escrita em um modelo que incluiu a apreensão e o autoconceito para escrita, o valor dado à tarefa e a realização anterior de escrita, a autoeficácia para autorregulação, o gênero e o nível de escolaridade. A autoeficácia para escrita explicou $49 \%$ da variabilidade da competência em escrita. Desse modo, mais uma vez comprovou-se o papel essencial das crenças de autoeficácia sobre a escrita.

A crença que os indivíduos possuem a respeito de suas próprias capacidades de planejar e implementar ações necessárias para alcançar objetivos de escritas em específicas tarefas também tem sido apontada como uma variável importante que interfere na escrita (Zimmerman \& Risemberg, 1997). Essa crença é denominada pela literatura de autoeficácia para autorregulação da escrita. De modo geral, autores salientam que esse construto se relaciona à maior competência em escrita, ao uso mais frequente de estratégias autorregulatórias, ao uso de uma abordagem profunda à aprendizagem, às atribuições de sucesso e fracasso mais adaptativas e à obtenção de sucesso acadêmico (Emilio, 2017; Limpo \& Alves, 2017; Shunck \& Zimmerman, 2007; Zimmerman \& Bandura, 1994; Zimmerman \& Kitsantas, 2002).

Zimmerman e Risemberg (1997) explicam que os escritores desenvolvem a percepção de autoeficácia para autorregulação da escrita à medida que notam quão bem-sucedidos foram nas produções textuais em decorrência do uso das estratégias autorregulatórias, embora seja possível sinalizar que as demais fontes de autoeficácia também podem impactar na percepção de autoeficácia. Da mesma forma, o uso de estratégias autorregulatórias desencadeia uma maior confiança para enfrentarem as atividades de escrita.

A fim de aprofundar o conhecimento sobre a percepção de autoeficácia para autorregulação da escrita e sua relação com a qualidade da escrita, Zimmerman e Bandura (1994) construíram uma escala para medição desse construto em uma amostra de universitários norte-americanos. A Escala de Autoeficácia para Autorregulação da Escrita (EAARE) avalia a percepção de capacidade do estudante em: a) executar aspectos estratégicos do processo de escrita, como o planejamento e a revisão; b) criativamente selecionar bons temas e escrever tópicos interessantes; c) regular o seu tempo e motivação. No estudo de validação, obteve-se uma solução unifatorial, cujo valor do alfa de Cronbach foi satisfatório $(\alpha=0,91)$.

No mesmo estudo, Zimmerman e Bandura (1994) investigaram as relações entre as crenças de autoeficácia para autorregulação da escrita, as crenças de autoeficácia para a realização acadêmica (crença na capacidade de dominar assuntos acadêmicos), os objetivos acadêmicos, os padrões pessoais para avaliação da qualidade da escrita, a aptidão verbal, o nível de instrução dos estudantes e a nota final em um curso sobre escrita. Os autores identificaram que as crenças de autoeficácia para autorregulação da escrita estavam direta e significativamente associadas com as crenças de autoeficácia para a realização acadêmica $(r=0,36 ; p<0,001)$, com os objetivos acadêmicos $(r=0,36 ; p<0,001)$ e com os padrões pessoais de avaliação $(r=0,39 ; p<0,001)$, mas não com a nota final do curso de escrita. Por meio de path analysis, esses autores observaram que as crenças de autoeficácia para autorregulação da escrita também não previram diretamente o desempenho final, mas previram as crenças de autoeficácia para a realização acadêmicas e os padrões autoavaliativos. Por sua vez, essas duas últimas variáveis influenciaram o desempenho final. Desse modo, os estudantes mais seguros em relação à sua capacidade de autorregular e expressar suas ideias por meio da escrita tiveram mais razão para acreditar em sua eficácia para dominar assuntos acadêmicos. Além disso, os estudantes que tiveram um alto senso de eficácia para gerenciar sua escrita, não somente definiram aspirações altas de escrita para si, mas estavam descontentes com desempenhos abaixo do padrão.

A crença de autoeficácia para autorregulação da escrita também foi objeto de estudo de Sanders-Reio (2010). A autora investigou as relações dessa variável 
com as crenças sobre como escrever, a percepção de apreensão em escrever e o desempenho na escrita de estudantes espanhóis. Os resultados indicaram uma correlação significativa, direta, porém fraca, entre autoeficácia para autorregulação da escrita e desempenho na escrita $(r=0,17 ; p<0,001)$. Sanders-Reio (2010) sugere que pesquisas futuras explorem relações de causa e efeito entre as variáveis, uma vez que o estudo utilizou somente análise correlacional.

Considerando a literatura brasileira, nota-se uma escassez de estudos sobre a autoeficácia e variáveis relacionadas à escrita desenvolvidos no contexto do Ensino Superior. Emilio (2017) localizou apenas o estudo de Veiga-Simão, Frison e Machado (2015) com essa temática. Supõe-se que essa escassez decorre, dentre outros motivos, da ausência de instrumentos validados para a população brasileira. Portanto, a tradução, a adaptação e validação da EAARE para o Brasil, poderá ser relevante para investigadores interessados na compreensão do processo de escrita e por profissionais de instituições de Ensino Superior que almejam identificar e auxiliar os estudantes a desenvolverem competências autorregulatórias da escrita

Os objetivos deste trabalho foram: 1 - Traduzir e adaptar a EAARE para a realidade brasileira; 2 Verificar a validade fatorial; 3 - Verificar o índice de consistência interna (alfa de Cronbach) da EAARE; 4 - Buscar evidência de validade convergente entre a EAARE e o Questionário de Crenças de Autoeficácia na Escrita (Villalón Molina, 2010). As hipóteses são de que a versão portuguesa/brasileira da EAARE também apresente um único fator (Zimmerman \& Bandura, 1994), índice de consistência interna satisfatório (Hair, Anderson, Tatham, \& Black, 2008) e de que haja uma correlação positiva entre os escores das duas escalas utilizadas (Reppold, Gurgel, \& Hutz, 2014).

\section{Método}

\section{Tradução e Adaptação da Escala}

Para a realização da tradução e adaptação da EAARE, foram realizadas quatro etapas distintas. Inicialmente, a EAARE (Zimmerman \& Bandura, 1994) foi traduzida do inglês norte-americano para o português brasileiro por dois pesquisadores bilíngues e familiarizados com o construto avaliado. Nessa etapa, cada pesquisador produziu independentemente uma versão do instrumento atentando-se para a preservação do significado e o sentido dos itens. Ambas traduções foram comparadas, demonstrando convergências, sendo realizados pequenos ajustes com a finalidade de se obter uma única versão inicial.

$\mathrm{Na}$ segunda etapa, a versão inicial da EAARE foi submetida à análise de seis juízes fluentes em inglês. Cada juiz recebeu a versão original (inglês) e a versão traduzida (português) e, então, avaliaram se a tradução dos itens estava ou não adequada. Quando inadequada, os juízes deveriam propor uma nova tradução ao item. Para todos os itens, a maioria dos juízes concordou com a correspondência de sentido entre a versão original e a versão traduzida, indicando a equivalência semântica (vocabulário e gramática) e conceitual (expressões com sentidos equivalentes).

$\mathrm{Na}$ terceira etapa, os apontamentos dos juízes foram averiguados por um comitê de especialistas, integrantes de um grupo de pesquisa que estuda o construto da autorregulação da aprendizagem e da autoeficácia, com a finalidade de consolidar a versão adaptada da escala. E, por fim, essa versão do instrumento foi aplicada em 15 estudantes voluntários do Ensino Superior de diferentes cursos e anos de formação. Os voluntários foram questionados a respeito de possíveis dificuldades encontradas nas instruções de preenchimento, nas escalas de respostas (que variava de 1 a 7 ) e nas expressões dos itens. Não foram constatadas dificuldades.

Sendo assim, a versão final da EAARE foi composta e sua tradução pode ser observada na Tabela 1. Essa versão foi aplicada nos participantes para as análises posteriores.

\section{Participantes}

Participaram da pesquisa 430 universitários de uma instituição pública de Ensino Superior localizada em uma cidade do estado de São Paulo, com idade média de 22,4 anos $(D P=4,8)$, sendo a maioria do sexo feminino $(54,9 \%)$, solteira $(93,7 \%)$ e não exercia trabalho remunerado $(61,9 \%)$. A área do curso predominante foi a de Ciências exatas e tecnológicas (36,9\%), seguida pela área de Ciências humanas (32,1\%), Ciências biológicas e da saúde $(22,3 \%)$ e Artes (8,6\%). Preponderaram estudantes que cursavam os semestres intermediários $(59,4 \%)$, sendo o restante de estudantes concluintes $(23,3 \%)$ e ingressantes (17,2\%). Quanto ao turno, 65,9\% estavam matriculados em cursos de tempo integral e $34,4 \%$ em cursos de tempo parcial.

Enfatiza-se que o número de participantes foi definido a partir da regra comumente utilizada em estudos das propriedades psicométricas de escalas, que sugere a necessidade de sete a dez sujeitos por item da escala (Freitas \& Borges-Andrade, 2004). No caso 
do presente estudo, seria preciso ter como sujeitos um número superior a 175, critério que foi atingido.

\section{Instrumentos}

O caderno de coleta de dados foi constituído por um questionário de caracterização, que visou obter dados de natureza pessoal (idade e sexo) e acadêmica (ano de formação e curso) dos participantes, e por dois instrumentos de autorrelato, sendo a EAARE traduzida e adaptada (Zimmerman \& Bandura, 1994), e o outro o Questionário de Crenças de Autoeficácia na Escrita (Villalón Molina, 2010).

O Questionário de Crenças de Autoeficácia na Escrita (QCAE) foi construído a partir da adaptação da escala de Shell et al. (1989) para o espanhol e elaboração de uma nova escala sobre tarefas de escrita a partir do trabalho de Solé et al. (2005). O QCAE avalia a confiança do aluno em suas habilidades como escritor e é constituído por duas subescalas, uma acerca das habilidades em escrita $(\alpha=0,79)$ e outra sobre a realização das tarefas de escrita $(\alpha=0,89)$. É composto por 22 itens que devem ser respondidos em uma escala de 0 a 100, sendo que o valor 0 corresponde à crença "não posso fazer", o valor 50 equivale à crença "sinto-me relativamente seguro em fazer" e o valor 100 remete à crença "seguro em fazer". Recentemente, esse instrumento foi adaptado e validado para o Brasil por Polydoro, Pelissoni, Dantas, Emilio e Carmo (2016). $\mathrm{Na}$ versão brasileira, manteve-se a escala de 0 a 100 pontos, todavia, após a realização de análises fatoriais exploratórias, optou-se pela organização unifatorial do questionário, que explicou $51,1 \%$ da variabilidade dos dados, obtidos junto aos 22 itens. Notou-se alta consistência interna para o instrumento $(\alpha=0,95)$.

\section{Procedimentos para Coleta de Dados}

Após aprovação do Comitê de Ética em Pesquisa (CEP), a coleta do estudo foi divulgada, via e-mail institucional, aos alunos matriculados em cursos de graduação da instituição participante. No e-mail havia informações sobre os objetivos da pesquisa, os procedimentos de coleta de dados e os princípios éticos que a nortearam. Havia também um link que direcionava os estudantes para o caderno de instrumentos em uma plataforma virtual de coleta de dados (Google docs), cuja página inicial continha as instruções gerais para o preenchimento dos instrumentos e informações sobre as condições de participação voluntária e a confidencialidade dos dados. O Termo de Consentimento Livre e Esclarecido (TCLE) foi apresentado na sequência, devendo o participante indicar seu consentimento em participar. Destaca-se que cada estudante recebeu um e-mail com cópia do TCLE para eventual consulta e contato com os pesquisadores ou o CEP. A participação no estudo se efetivava quando o estudante respondia aos instrumentos e clicava em "enviar" as respostas. O tempo para o preenchimento dos instrumentos foi de aproximadamente 30 minutos.

\section{Análises dos Dados}

O processo de validação da EAARE abrangeu duas etapas gerais: 1) Busca por evidências de validade baseadas na estrutura interna e 2) Busca por evidências de validade baseadas na relação com variáveis externas convergentes (Reppold et al., 2014). Nesse sentido, a primeira etapa teve por foco as propriedades psicométricas da EAARE, abarcando análises fatoriais exploratórias e análises da consistência interna (alfa de Cronbach). Para se verificar a composição fatorial, realizou-se, inicialmente, a análise dos níveis de Kaiser-Meyer-Olkin (KMO) e o teste de esfericidade de Bartlett. Para o teste dos modelos, usou-se a extração dos eixos principais PAF (Principal Axis Factoring). A primeira rotação utilizada foi a Varimax, todavia a rotação Promax também foi conduzida, já que permite que os fatores se correlacionem, demonstrando ser a mais adequada. Na segunda etapa, a validade foi testada a partir das correlações de Pearson estabelecidas entre os escores da escala testada e os indicadores externos (escore de um instrumento convergente, no caso o QCAE).

\section{Resultados}

\section{Evidências de Validade da EAARE Baseadas na Estrutura Interna}

Nas análises preliminares, examinou-se o atendimento das condições necessárias para a realização da análise fatorial. $O$ índice de confiança da análise $(\mathrm{KMO}=0,95)$ foi satisfatório e o teste de esfericidade de Bartlett $\left(\chi^{2}=6399.27 ; p<0,001\right)$ foi significativo, indicando, respectivamente, que as matrizes de dados da EAARE eram passíveis de fatoração. Mediante a análise fatorial exploratória (AFE), os resultados indicaram a presença de quatro fatores, cujos autovalores (eigenvalue) eram superiores a 1 e responsáveis pela explicação de $61,4 \%$ da variabilidade dos dados. Todavia, pelo teste do scree plot (Figura 1), optou-se por fixar a extração de três fatores, que explicaram 56,9\% da variabilidade total, uma vez que, a partir do terceiro fator, a curva se estabilizava. 
A primeira rotação utilizada foi a Varimax para três fatores. O Fator 1 foi composto por 12 itens; o Fator 2 por nove itens; o Fator 3 por quatro itens. Todos os itens apresentaram carga fatorial satisfatória (superior a 0,30 ) e vários deles tiveram cargas altas em mais de um fator e, por isso, foram alocados no fator de maior carga. Também foi utilizada a rotação oblíqua Promax para estudar a composição dos fatores, supondo haver correlação entre eles. Semelhantemente à primeira análise, notou-se que o Fator 1 foi composto por 12 itens com carga maior que 0,30 (Itens: 2, 3, 4, 1, 8, 5, 16, 19, 6, 20, 11 e 17); o segundo, por nove itens (Itens: 21, 23, $22,12,13,7,15,10$ e 25$)$ e o terceiro, por quatro itens (Itens: 24, 18, 14, e 9). Alguns itens apresentaram cargas altas em mais de um fator e, dessa forma, foram colocados no fator de carga mais elevada. Dada a correlação significativa e positiva entre os três fatores, a rotação Promax se mostrou mais adequada.

A confiabilidade da escala pelos fatores obtidos nessa AFE indicou consistência interna satisfatória para os três fatores e para o total da escala. O primeiro e o segundo fatores apresentaram um índice de confiabilidade de 0,91, enquanto o terceiro teve o alfa de Cronbach de 0,70. Ainda que a versão EAARE com três fatores tenha apresentado bons índices psicométricos, notou-se que os agrupamentos dos itens nos fatores não eram coerentes entre si, por exemplo: o item 16 (Sou capaz de reorientar a minha concentração na escrita quando me vejo pensando em outras coisas) e o item quatro (Sou capaz de criar um parágrafo inicial que desperte o interesse dos leitores) estavam agrupados no mesmo fator, mas um avalia a percepção de

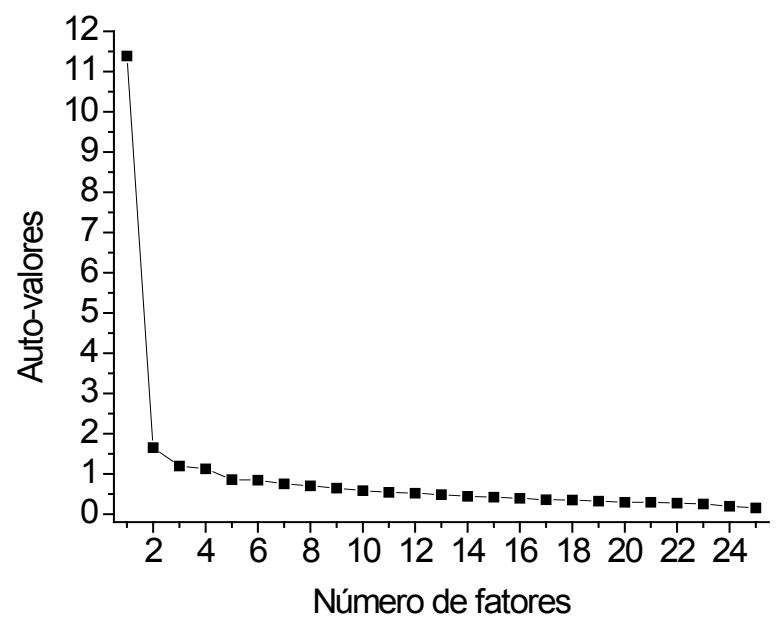

Figura 1. Teste do scree plot para número de fatores da escala. capacidade de manter a concentração e o outro avalia a percepção de capacidade de escrever criativamente uma introdução.

Em virtude desse tipo problema e, também, de a escala original apresentar um único fator, a AFE foi refeita considerando apenas um fator. O fator explicou $45,6 \%$ da variabilidade dos dados e foi composto por 25 itens com carga maior que 0,30 (Tabela 1). A confiabilidade da escala pelo fator obtido na AFE foi analisada e notou-se consistência interna satisfatória $(0,95)$.

Este trabalho optou pelo uso da estrutura unifatorial da EAARE, pois esta, além de replicar a versão original do instrumento de Zimmerman e Bandura (1994), é considerada pelos estudos psicométricos da AFE e de consistência interna como um instrumento válido, fidedigno e próprio para o uso no contexto nacional. Ademais, atende ao critério parcimônia.

\section{Evidência de Validade Baseada na Relação com Variável Externa Convergente}

Com o intuito de encontrar evidência de validade convergente, submeteram-se os escores da EAARE e do Questionário de Crenças de Autoeficácia na Escrita (QCAE) à análise de correlação de Pearson. Os resultados apontaram uma relação estatisticamente significativa, forte e positiva $(r=0,70 ; p<0,001)$ entre os escores médios totais das duas escalas, indicando que os participantes que se avaliaram como mais eficazes para autorregular os aspectos cognitivos, motivacionais e comportamentais envolvidos no processo de escrita tenderam também a se perceber mais capazes para realização de diferentes tarefas de escrita e capazes em habilidades em escrita e vice e versa.

\section{Discussão}

Reportando-se aos processos de tradução e de adaptação cultural da EAARE, estes se mostraram eficazes para com os objetivos do estudo. Foi adotada uma abordagem com diversas etapas (tradução; síntese; revisão da primeira versão; análise dos apontamentos dos juízes por um comitê de especialistas; submissão do instrumento a leigos; revisão e consolidação da versão final da escala) que garantiu a equivalência conceitual e a fácil compreensão dos indivíduos que preencheram a escala.

Nos estudos de validação da escala original, os autores Zimmerman e Bandura (1994) utilizaram procedimentos de componentes principais e selecionaram uma solução de fator que abrangeu todos os 
Tabela 1.

Cargas Fatoriais dos Itens na Proposta Unifatorial da EAARE Adaptada

\begin{tabular}{ccc}
\hline & Itens & Fator 1
\end{tabular}

15 Quanto eu sou capaz de escrever a transição de uma ideia para outra, utilizando frases eficazes? 0,80

5 Quanto eu sou capaz de escrever uma apresentação geral breve e informativa, que irá preparar os $\quad 0,76$ leitores para a ideia principal do meu texto?

17 Quando escrevo um texto longo, quanto eu sou capaz de criar uma variedade de bons tópicos para as 0,76 seções principais do meu texto?

13 Quando eu preciso escrever uma ideia abstrata de forma mais clara, quanto eu sou capaz de usar 0,76 palavras para criar uma imagem nítida?

10 Quanto eu sou capaz de atender aos padrões de escrita de um avaliador muito exigente? 0,75

4 Quanto eu sou capaz de criar um parágrafo inicial que desperte o interesse dos leitores?

12 Quanto eu sou capaz de reescrever as frases longas ou confusas do meu texto para que fiquem claras? 0,74

22 Quanto eu sou capaz de revisar um primeiro esboço de qualquer texto para que ele fique mais curto e 0,72 melhor organizado?

3 Quanto eu sou capaz de construir rapidamente uma boa frase de introdução? 0,72

1 Diante de uma tarefa específica de escrita, quanto eu sou capaz de escrever de forma apropriada sobre $\quad 0,71$ o tema em um curto espaço de tempo?

19 Quando fico sem ideias ao escrever um texto, quanto eu sou capaz de encontrar maneiras de superar o $\quad 0,70$ problema?

2 Quanto eu sou capaz de começar a escrever um texto sem dificuldade? $\quad 0,70$

6 Quanto eu sou capaz de usar minhas primeiras tentativas de escrita para refinar minhas ideias sobre $\quad 0,69$ um tema?

7 Quanto eu sou capaz de ajustar meu estilo de escrita para atender às necessidades de diferentes $\quad 0,68$ públicos?

11 Quanto eu sou capaz de rapidamente criar bons exemplos para ilustrar um ponto importante do texto? 0,67

16 Quanto eu sou capaz de reorientar a minha concentração na escrita quando me vejo pensando em 0,64 outras coisas?

25 Quando escrevo sobre um tema complicado, quanto eu sou capaz de apresentar um título curto e 0,64 informativo?

14 Quanto eu sou capaz de localizar e usar fontes de referência apropriadas quando preciso fundamentar $\quad 0,63$ um ponto importante no texto?

21 Quando eu escrevo um texto longo ou complexo, quanto eu sou capaz de encontrar e corrigir os erros 0,61 gramaticais?

18 Quando eu quero convencer um leitor sobre um ponto, quanto eu sou capaz de utilizar uma citação convincente de uma autoridade no assunto?

23 Quando eu reviso um texto complexo, quanto eu sou capaz de encontrar e corrigir todos os meus erros gramaticais?

20 Quanto eu sou capaz de encontrar maneiras de me motivar a escrever um texto, mesmo quando o tema tem pouco interesse para mim?

8 Quanto eu sou capaz de encontrar uma maneira de me concentrar na escrita do texto, mesmo quando 0,55 existem muitas distrações ao meu redor?

9 Quanto eu sou capaz de gerenciar meu tempo eficientemente, mesmo quando tenho pressão de um 0,55 prazo para entregar um texto?

24 Quanto eu sou capaz de encontrar pessoas que darão feedback sobre os primeiros esboços do meu texto? 
itens, com exceção dos itens 9 e 20, sendo o valor do alfa de Cronbach de 0,91. No caso da presente pesquisa, empregou-se procedimentos de análise fatorial exploratória e optou-se por uma estrutura unifatorial que compreendeu os 25 itens. Figueiredo Filho e Silva Júnior (2010) elucidam que ambas as técnicas - a análise de componentes principais e a análise fatorial buscam produzir combinações lineares de variáveis que capturem o máximo possível de variância das variáveis observadas. $\mathrm{Na}$ primeira, toda a variância é utilizada. Por sua vez, na análise fatorial, somente a variância compartilhada (Dancey \& Reidy, 2006). Além disso, a análise de componentes principais é preferida quando se busca um resumo empírico do conjunto de dados, enquanto a análise fatorial é a melhor opção quando se está interessado em uma solução teórica não contaminada por variabilidade de erro. Apesar dessas distinções, Hair, Anderson, Tatham e Black (2008) dizem que, na maioria dos casos, as duas técnicas chegam a resultados semelhantes.

A validade de um instrumento também pode ser atestada quando as correlações entre os escores da escala testada e os indicadores de outra medida assumem magnitude e direção coerentes com as expectativas formuladas com base na literatura (Reppold, Gurgel \& Hutz, 2015; Urbina, 2007). Nesse sentido, a relação estatisticamente significativa, forte e direta entre os escores médios da EAARE e da QCAE, endossou que a EAARE é um instrumento psicometricamente adequado para uso nacional.

Frente às evidências de validade e de confiabilidade da EAARE, o presente estudo recomenda o uso da versão brasileira constituída por 25 itens. Estes devem ser respondidos em uma escala Likert de sete pontos, sendo que o ponto 1 equivale à crença de que não é capaz de realizar as atividades designadas, e o valor 7 à crença de que é muito capaz de realizá-la. O índice total é obtido a partir da somatória de todos os itens, divididos por 25. A estrutura de pontuação da EAARE considera que, quanto menor o índice obtido, menor é a percepção de autoeficácia para autorregulação da escrita, e que quanto maior o índice obtido, maior é a percepção de autoeficácia para autorregulação da escrita.

Apesar das crenças de autoeficácia já serem objeto de análise de vários estudos científicos, tal como nos estudos de Polydoro e Guerreiro-Casanova (2010) sobre autoeficácia para formação superior, de Emilio e Polydoro (2017) sobre autoeficácia para autorregulação da aprendizagem e de Torisu e Ferreira (2009) sobre autoeficácia matemática, dentre outros, nenhum estudo nacional com o escopo na autoeficácia para a autorregulação da escrita foi encontrado. Nesse sentido, a validação da EAARE para o Brasil poderá abrir novos caminhos de investigação.

\section{Considerações Finais}

A maneira como os estudantes percebem e desenvolvem a sua capacidade de escrita frente às exigências do Ensino Superior tem um grande impacto no sucesso desses estudantes, pois eles passam grande parte do tempo a reter informações que lhes são apresentadas sob a forma de textos e, posteriormente, precisam expor, por meio da escrita, o conhecimento adquirido. Muito se observa que os professores tomam as produções textuais como indicativos para avaliar o quanto o estudante dominou os conteúdos de suas disciplinas. Mateos, Villalón, Dios e Martín (2007) acrescentam que a escrita tem um papel central no Ensino Superior, pois é uma ferramenta epistemológica que possibilita a aprendizagem de um conteúdo - identificando ideias, comparando-as, sintetizando-as e organizando-as em um texto -, bem como a transformação e a produção de novos conhecimentos, já que os argumentos usados na escrita podem provocar modificações de opinião e de valores, além da ampliação de conhecimentos de quem escreve e de quem lê.

Dado que a EAARE mensura a crença de autoeficácia para autorregular fatores importantes envolvidos no processo de escrita, tais como: regulação da motivação, planejamento, escrita da introdução, execução da revisão, gestão do tempo etc., as possibilidades de aplicação mostram-se diversificadas no âmbito investigativo e/ ou prático. Ela poderá ser utilizada por pesquisadores interessados na compreensão do processo de escrita e por profissionais de instituições de educação superior que almejam identificar e auxiliar os estudantes a desenvolverem competências autorregulatórias da escrita.

Adverte-se que o processo de validação contou com uma amostra de estudantes universitários de uma única instituição pública do estado de São Paulo, a qual, embora possa abranger participantes de idades variadas e de diferentes condições socioeconômicas, ainda implica em uma restrição na generalização dos resultados para a população brasileira e para população de estudantes do Ensino Superior. Portanto, sugere-se que estudos futuros examinem se as qualidades psicométricas obtidas são equivalentes em amostras diversificadas em termos de região (estudantes de outros estados), 
de nível acadêmico (estudantes de pós-graduação, por exemplo), de tipo de instituição (privadas e públicas) e de modalidades de ensino (presencial e a distância). Também seria interessante a realização de estudos posteriores com outros tipos de validade e confiabilidade (Menescal, 2018; Wolter \& Won, 2018).

\section{Referências}

Balkis, M., \& Duru, E. (2016). Procrastination, selfregulation failure, academic life satisfaction, and affective well-being: Underregulation or misregulation form. European Journal of Psychology of Education, 31(3), 439-459. doi:10.17583/ijep.2017.2584

Carmo, M. C. (2017) Avaliação de um programa de intervenção na escrita de sinteses para estudantes universitários (Tese de doutorado). Faculdade de Educação, Universidade Estadual de Campinas, Campinas, Brasil. Recuperado de http://www.repositorio.unicamp. $\mathrm{br} /$ handle/REPOSIP/330987

Dancey, C., \& Reidy, J. (2006). Estatística sem matemática para psicologia. Porto Alegre: Artmed.

Emilio, E. R. V. (2017). Autorregulação, Autoeficácia, abordagens a aprendizagem e a escrita de universitários (Tese de doutorado). Faculdade de Educação, Universidade Estadual de Campinas, Campinas, Brasil. Recuperado de http://www.repositorio.unicamp. $\mathrm{br} /$ handle/REPOSIP/322738

Emilio, E. R. V., \& Polydoro, S. A. J. (2017). Autorregulação da aprendizagem: fundamentos e implicações no contexto educativo. Em S. A. J. Polydoro (Eds.). Promoção da autorregulação da aprendizagem: contribuições da teoria social cognitiva. (1 ${ }^{a}$ ed., pp. 19-31). Porto Alegre: Letra1.

Fernandes, V. R., \& Frison, L. M. B. (2015). Estratégias de aprendizagem autorregulatória no ensino superior: Escrita de um artigo científico. Psicologia da Educação, 41, 37-49. doi: 10.5935/2175-3520.20150013

Figueiredo Filho, D. B., \& Silva Júnior, J. A. D. (2010). Visão além do alcance: Uma introdução à análise fatorial. Opinião Pública, 16(1), 160-185. doi: 10.1590/S0104-62762010000100007

Freitas, I. A., \& Borges-Andrade, J. E. (2004). Construção e validação de Escala de Crenças sobre o Sistema Treinamento. Estudos de Psicologia, 9(3), 479488. doi:10.1590/S1413-294X2004000300010.
Ganda, D. R., \& Boruchovitch, E. (2018). A autorregulação da aprendizagem: Principais conceitos e modelos teóricos. Psicologia da Educação, (46), 71-80. Recuperado de https://revistas.pucsp.br/ psicoeduca/article/view/39147

Granam, S, Harris, K. R., MacArthur, C., \& Santangelo, T. (2018). Self-regulation and writing. Em D. H. Schunk, \& J. A. Greene (Eds.). Handbook of selfregulation of learning and performance. (pp. 138-152). New York: Routledge.

Grau, V., \& Whitebread, D. (2012). Self and social regulation of learning during collaborative activities in the classroom: The interplay of individual and group cognition. Learning and Instruction, 22(6), 401412. doi:10.1016/j.learninstruc.2012.03.003

Hair, F., Anderson, R. E., Tatham, R. L., \& Black, W. C. (2008). Análise multivariada de dados. (S. S. Adonai, \& C. N. Anselmo, Trads. (5 ${ }^{\mathrm{a}}$ ed.). Porto Alegre: Bookman.

Herndon, J. S., \& Bembenutty, H. (2017). Self-regulation of learning and performance among students enrolled in a disciplinary alternative school. Personality and individual differences, 104, 266-271. doi:10.1016/j. paid.2016.08.027

Limpo, T., \& Alves, R. A. (2017). Relating beliefs in writing skill malleability to writing performance: The mediating role of achievement goals and selfefficacy. Journal of Writing Research, 9(2), 97-125. doi:10.17239/jowr-2017.09.02.01

Marini, J. A. S., \& Boruchovitch, E. (2014). Estratégias de aprendizagem de alunos brasileiros do ensino superior: Considerações sobre adaptação, sucesso acadêmico e aprendizagem autorregulada. Revista E-Psi, 4(1), 102-126. Recuperado de https:/ / revistaepsi.com/wp-content/uploads/artigos/2014/ Ano4-Volume1-Artigo5.pdf

Mateos, M., Villalón, R., Dios, M. J., \& Martín, E. (2007). Reading and writing tasks on different university degree courses: What do the students say they do? Studies in Higher Education, 32(4), 489-510. doi:10.1080/03075070701476183

McCarthy, P., Meier, S., \& Rinderer, R. (1985). Self-efficacy and writing: A different view of self-evaluation. College composition and communication, 36(4), 465-471. doi:10.2307/357865 
Mello, A. S. L., Rosário, P. S. L. F., Polydoro, S. A. J., \& Högemann, J. H. (2018). Ansiedade reportada frente à produção textual: Correlações com variáveis motivacionais. Revista de Educação PUC-Campinas, 23(3), 407-424. doi:10.24220/2318-0870v23n3a4326

Menescal, N. R. G. (2018). Instrumentos de aferição da autorregulação da aprendizagem em universitários (Tese de doutorado). Instituto de Psicologia, Universidade de São Paulo, São Paulo, Brasil. doi:10.11606/T.47.2018.tde-27072018-104844

Nava, M. R. S. (2013). A escrita no Ensino Superior: contribuições da literatura (Dissertação de mestrado). Faculdade de Educação, Universidade Estadual de Campinas, Campinas, Brasil. Recuperado de http://repositorio.unicamp.br/jspui/handle/ REPOSIP/250771

Nava, M. R. S. (2017). A escrita como ferramenta epistêmica. Em S. A. J. Polydoro (Org.), Promoção da Autorregulação da Aprendizagem: Contribuições da Teoria Social Cognitiva (1 ${ }^{\mathrm{a}}$ ed., pp. 121-132). Porto Alegre: Letra1.

Nietto, R. F.S., \& ALVES, L. K. (2007). Reflexões sobre (re)escrita no ensino superior: Qual o papel do professor nesse contexto? Revista Lingua e Literatura, 9(13), 29-51. Recuperado de http://revistas. fw.uri.br/index.php/revistalinguaeliteratura/ article/view/67

Pajares, F., \& Johnson, M. J. (1996). Self-efficacy beliefs and the writing performance of entering high school students. Psychology in the Schools, 33(2), 163-175. doi:10.1002/(SICI)15206807(199604)33:2<163::AID-PITS10>3.0.CO;2-C

Pajares, F., \& Johnson, M. J. (1994). Confidence and competence in writing: The role of self-efficacy, outcome expectancy, and apprehension. Research in the Teaching of English, 313-331. Recupe rado de https://files.eric.ed.gov/fulltext/ED358474.pdf

Pajares, F., Johnson, M. J., \& Usher, E. L. (2007). Sources of writing self-efficacy beliefs of elementary, middle, and high school students. Research in the Teaching of English. 104-120. Recuperado de www. jstor.org/stable/40171749

Pajares, F., \& Valiante, G. (1999). Grade level and gender differences in the writing self-beliefs of middle school students. Contemporary Educational Psychology, 24(4), 390-405. doi:10.1006/ceps.1998.0995
Perassinoto, M., Boruchovitch, E., \& Bzuneck, J. A. (2013). Estratégias de aprendizagem e motivação para aprender de alunos do Ensino Fundamental. Avaliação Psicológica, 12(3), 351-359. Recuperado de http://pepsic.bvsalud.org/scielo.php?script=sci_ arttext\&pid=S1677-04712013000300010\&lng $=$ pt \&tlng $=\mathrm{pt}$

Polydoro, S. A. J, \& Guerreiro-Casanova D. C. (2010) Escala de autoeficácia na formação superior: construção e estudo de validação. Avaliação Psicológica, 9(2), 267-278. Recuperado de http:// pepsic.bvsalud.org/scielo.php?script $=$ sci_ arttext\&pid $=$ S1677-04712010000200011\&lng=pt \&tlng $=\mathrm{pt}$

Polydoro, S. A. J., Pelissoni, M. A. S., Dantas, M. A., Emilio, E. R. V., \& Carmo, M. C. (2016). Questionário de crenças de autoeficácia na escrita: Adaptação e validação. Em: I Seminário Internacional Aprendizagem Autorregulada e Motivação, Campinas, São Paulo, Brasil, pp. 199-200. Recuperado de https://www. fe.unicamp.br/eventos/aprendizagem-autorregulada/arquivos/anais.pdf

Polydoro, S. A. J., \& Emilio, E. R. V. (2017). Autoeficácia e autorregulação: Interfaces no âmbito da escrita. Em S. A. J. Polydoro (Eds.). Promoção da Autorregulação da Aprendizagem: Contribuições da Teoria Social Cognitiva (1 ${ }^{\text {a }}$ ed., pp. 111-125). Porto Alegre: Letra1.

Polydoro, S. A. J, Emilio, E. R. V., \& Carmo, M. C. (2017). Intervenções em Autorregulação da Escrita no Ensino Superior. Em S. A. J. Polydoro (Eds.). Promoção da Autorregulação da Aprendizagem: Contribuiçoes da Teoria Social Cognitiva (1ª ed., pp. 149-161). Porto Alegre: Letra1.

Polydoro, S. A. J., \& Pedersen, S. A. (2017). Programas de intervenção em Autorregulação da Escrita. Em S. A. J. Polydoro (Eds.). Promoção da Autorregulação da Aprendizagem: Contribuicões da Teoria Social Cognitiva (1 $1^{\mathrm{a}}$ ed., pp. 133-148). Porto Alegre: Letra1.

Pozo, J. (1996). Estratégias de aprendizagem. Em C. Coll, J. Palácios \& A. Marchesi (Eds.). Desenvolvimento psicológico e educação: psicologia da educaşão (p. 176-197). Porto Alegre: Artes Médicas.

Ramdass, D., \& Zimmerman, B. J. (2011). Developing self-regulation skills: The important role of homework. Journal of Advanced Academics, 22(2), 194-218. 
Reppold, C. T., Gurgel, L. G., \& Hutz, C. S. (2014). El proceso de construcción delas escalas psicométricas. Avaliação Psicológica, 13(2), 307-310. Recuperado de http://pepsic.bvsalud.org/pdf/avp/v13n2/ v13n2a18.pdf

Sanders-Reio, J. (2010). Investigation of the relations between domain-specific beliefs about writing, writing self-efficacy, writing apprehension, and writing performance in undergraduates (Tese de doutorado) Faculty of the Graduate School of the University of Mayland, Mayland, United States. Retrieved from http:// hdl.handle.net/1903/10249

Schunk, D. H., \& Greene, J. A. (2018). Historical, contemporary, and future perspectivas on selfregulated leraning and performance. Em D. H. Schunk, \& Greene, J. A. (Eds.). Handbook of selfregulation of learning and performance (pp. 01-16). New York: Routledge.

Schunk, D. H., \& Zimmerman, B. J. (2007). Influencing Children's Self-Efficacy and Self-Regulation of Reading and Writing Through. Reading \& Writing. Quarterly, 23,7-25. doi:10.1080/10573560600837578

Schunk, D. H., \& Zimmerman, B. J. (1997). Social origins of self-regulatory competence. Educational psychologist, 32(4), 195-208. doi:10.1207/ s15326985ep3204_1

Shell, D. F., Murphy, C. C., \& Bruning, R. H (1989). Self-efficacy and outcome expectancy mechanisms in reading and writing achievement. Journal of Educational Psychology, 81(1), 91-100. doi:10.1037/0022-0663.81.1.91

Solé, I., Mateos, M., Miras, M., Martín, E., Castells, N., Cuevas, I., \& Grácia, M. (2005). Lectura, escritura y adquisición de conocimientos en Educación Secundaria y Educación Universitaria. Infancia y Aprendizaje, 28(3), 329-348. Recuperado de https://dialnet.unirioja.es/servlet/ articulo?codigo $=1975628$

Thiede, K. W., \& Bruin, A. B. N. (2018). Self-regulated learning in reading. Em D. H. Schunk, \& J. A. Greene (Eds.). Handbook of self-regulation of learning andperformance (pp. 124-137). New York: Routledge.

Torisu, E. M., \& Ferreira, A. C. (2009). A Teoria Social Cognitiva e o ensino-aprendizagem da matemática: Considerações sobre as crenças de autoeficácia matemática. Ciências \&o cognição, 14(3), 168-177.
Recuperado de http://www.repositorio.ufop.br/ handle/123456789/4658

Urbina, S. (2007). Fundamentos da testagem psicológica. Porto Alegre: Artmed.

Veiga-Simão, A. M. D., Frison, L. M. B., \& Machado, R. F. (2015). Escrita de resumos e estratégias de autorregulação da aprendizagem. Cadernos de pesquisa, 45(155), 30-55. Recuperado de https:// www.scielo.br/pdf/cp/v45n155/1980-5314cp-45-155-00030.pdf

Villalon Molina, R. (2010). Las concepciones de los Estudiantes sobre la escritura acadêmica. (Tese de doutorado). Faculdade de Psicologia, Universidade Autonoma de Madri, Espanha. Recuperado de https://repositorio.uam.es/bitstream/handle/10486/4865/31711_villalon_ruth.pdf?sequ

Volet, S., Vauras, M., \& Salonen, P. (2009). Self-and social regulation in learning contexts: An integrative perspective. Educational psychologist, 44(4), 215-226. doi: 10.1080/00461520903213584

Weiner, B. (2010). The development of an attribution-based theory of motivation: A history of ideas. Educational Psychologist, 45(1), 28-36. doi:10.1080/00461520903433596

Wolters, C. A. (2011). Regulation of motivation: Contextual and social aspects. Teachers College Record, 113, 265-283. Recuperado de https://eric. ed.gov/?id=EJ 927078

Wolter, C. A., \& Won, S. (2018). Validity and the use of self-report questionnaires to asses self-regulated leraning. Em D. H. Schunk, \& J. A. Greene (Eds.). Handbook of self-regulation of learning and performance (pp. 307-322). New York: Routledge.

Zimmerman, B. J., \& Bandura, A. (1994). Impact of self-regulatory influences on writing course attainment. American Educacional Reseaarch Journal Winter, 31(4), 845-862. doi:10.3102/00028312031004845

Zimmerman, B. J., \& Kitsantas, A. (2002). Acquiring writing revision and self-regulatory skill through observation and emulation. Journal of Educational Psychology, 94(4), 660- 668. doi:10.1037/0022-0663.94.4.660

Zimmerman, B. J., \& Kitsantas, A. (2014). Comparing students' self-discipline and self-regulation measures and their prediction of academic achievement. 
Contemporary Educational Psychology, 39(2), 145-155. doi:10.1016/j.cedpsych.2014.03.004

Zimmerman, B. J., \& Schunk, D H. (Eds.). (2011). Educational psychology handbook series. Handbook of self-regulation of learning and performance. New York: Routledge/Taylor \& Francis Group.
Zimmerman, B. J., \& Risemberg, R. (1997). Become a self-regulated writer: A social Cognitive perspective. Contemporary Educational Psychology, 22(1), 73-101. doi:10.1006/ceps.1997.0919

Recebido em: 09/01/2019

Reformulado em: 20/11/2019

Aprovado em: 03/12/2019

Sobre as autoras:

Eduarla Resende Videira Emilio é Psicóloga, mestre em Psicologia da Saúde e doutora em Educação pela Universidade Estadual de Campinas. Atualmente é adjunta da Divisão de Pesquisa e Desenvolvimento do Instituto de Psicologia da Aeronáutica - IPA.

ORCID: https://orcid.org/0000-0003-2460-9016

E-mail: eduarla@uol.com.br

Soely Aparecida Jorge Polydoro é Psicóloga, mestre em Psicologia Escolar e doutora em Educação. Atualmente é Professora Doutora do departamento de Psicologia Educacional e do programa de pós-graduação em Educação, Faculdade de Educação da Universidade Estadual de Campinas, integrante do Grupo de Pesquisa Psicologia e Educação Superior, Coordenadora Geral do Espaço de Apoio ao Ensino e a Aprendizagem órgão da Pró-Reitoria de graduação.

ORCID: https://orcid.org/0000-0003-4823-3228

E-mail: soelypolydoro@gmail.com

Adriane Martins Soares Pelissoni é Pedagoga, mestre e doutora em Psicologia Educacional pelo programa de pós-graduação da Faculdade de Educação da Unicamp. Atualmente é supervisora acadêmica e orientadora educacional do Serviço de Apoio ao Estudante - SAE/Universidade Estadual de Campinas - UNICAMP.

ORCID: https://orcid.org/0000-0002-8850-5569

E-mail: adrianepelissoni@gmail.com

Marilda Aparecida Dantas é Pedagoga e Psicóloga. Mestre em Avaliação Psicológica. Doutora em Psicologia Educacional pelo programa de pós-graduação da Faculdade de Educação da Unicamp. Atualmente é orientadora educacional do Serviço de Apoio ao Estudante - SAE/Universidade Estadual de Campinas - UNICAMP

ORCID: https:/ / orcid.org/0000-0001-6858-6421

E-mail:marildapsi@uol.com

Contato com as autoras:

Faculdade de Educação da Unicamp

Av. Bertrand Russell, 801, Cidade Universitária "Zeferino Vaz"

Campinas-SP, Brasil

CEP: $13083-865$ 